\title{
ESTUDO DA INFLUÊNCIA DA VAZÃO DE INJEÇÃO DE VAPOR NO PROCESSO ES-SAGD SEM E COM PERDA DE CARGA E CALOR NO POÇO INJETOR
}

\author{
T. S. PRAXEDES ${ }^{1}$, J. L. M. BARILLAS ${ }^{2}$ \\ ${ }^{1}$ Universidade Federal do Rio Grande do Norte, Programa de Pós Graduação em Ciência e \\ Engenharia de Petróleo \\ ${ }^{2}$ Universidade Federal do Rio Grande do Norte, Programa de Pós Graduação em Ciência e \\ Engenharia de Petróleo \\ E-mail para contato: taysuelly@ yahoo.com.br
}

RESUMO - O processo de drenagem gravitacional assistida com vapor e solvente (ESSAGD) faz uso de uma tecnologia promissora para recuperação de óleos pesados. Ele utiliza dois poços horizontais paralelos, onde o injetor é disposto acima do produtor. No poço injetor ocorre a co-injeção de vapor e solvente que aquece o óleo impulsionando-o por gravidade até o poço produtor. Neste estudo foi utilizado o modelo discretizado, onde o poço é discretizado da mesma forma que o reservatório, sendo cada seção do poço tratada como um bloco da malha, com conexão interblocos com o reservatório. O presente trabalho tem como objetivo analisar a influência da vazão de injeção de vapor no processo ES-SAGD quando se considera e desconsidera a perda de carga e calor no poço injetor. $\mathrm{O}$ modelo utilizado para estudo trata-se de um reservatório homogêneo, semissintético com características do Nordeste Brasileiro e as simulações numéricas foram realizadas através do simulador térmico STARS da CMG (Computer Modelling Group). Os resultados demonstraram que, o modelo que considera a perda de carga e calor apresenta produção acumulada de óleo inferior ao seu respectivo modelo que desconsidera tais perdas. $\mathrm{O}$ modelo com vazão de injeção de vapor baixa gera maior dificuldade no aquecimento e produção do óleo presente no reservatório.

\section{INTRODUÇÃO}

O processo ES-SAGD consiste na adição de uma pequena quantidade de solvente com vapor no processo SAGD (Deng et al., 2011). Estes processos utilizam dois poços horizontais paralelos separados por certa distância vertical, onde o poço superior é o injetor e o inferior é o produtor (Barillas, 2005).

O poço superior recebe continuamente o vapor-solvente e injeta-o no reservatório, o óleo é aquecido e forma uma câmara de vapor em torno dos poços que cresce para cima e para os arredores (Butler, 1991). O solvente cujo comportamento termodinâmico de vaporização é semelhante, ou próximo, ao comportamento termodinâmico da água para uma determinada condição de reservatório é considerado como o mais apropriado para se injetado junto com o vapor (Nasr e Ayodele, 2006). 
O vapor contribui com calor para redução da viscosidade do óleo e o solvente ajuda na miscibilidade, reduzindo a tensão interfacial entre óleo/solvente. Na interface com o óleo frio o vapor transfere calor ao óleo e condensa. Por fim, o óleo quente e a água condensada drenam por gravidade até o produtor horizontal inferior. A principal força atuante neste processo é a gravitacional.

A câmara de vapor cresce verticalmente tendo como limite o topo do reservatório e se estende horizontalmente até atingir os limites laterais do reservatório. O espaço antes ocupado pelo óleo é substituído pelo vapor. Esse esquema propicia a drenagem de grande área do reservatório.

Neste estudo os poços horizontais foram modelados através do modelo discretizado, onde são considerados o escoamento do fluido e o calor no poço. Neste modelo o poço é discretizado da mesma forma que o reservatório, sendo cada seção do poço tratada como um bloco da malha, com conexão interblocos com o reservatório.

Este trabalho tem por objetivo analisar a influência que a vazão de injeção de vapor exerce sobre o processo ES-SAGD quando se considera e desconsidera a perda de carga e calor no poço injetor.

\section{METODOLOGIA}

Neste estudo foram realizadas simulações e posteriores análises gráficas dos resultados obtidos.

Foi analisado um reservatório homogêneo com características do Nordeste Brasileiro que possui as seguintes dimensões $100 \mathrm{~m}$ x $600 \mathrm{~m}$ x $26 \mathrm{~m}$, o número total de blocos na malha 27 × 30 × $26 . \mathrm{O}$ comprimento dos poços horizontais é de $500 \mathrm{~m}$ cada e o tempo de simulação foi baseado num período de 20 anos. As características do reservatório podem ser observadas na Tabela 1.

Tabela 1 - Propriedades do reservatório

\begin{tabular}{|c|c|}
\hline Propriedade & Valor \\
\hline Porosidade (\%) & 30 \\
\hline $\begin{array}{c}\text { Permeabilidade } \\
\text { horizontal, Kh (mD) }\end{array}$ & 1000 \\
\hline $\begin{array}{c}\text { Permeabilidade } \\
\text { vertical, Kv (mD) }\end{array}$ & $0.1 * \mathrm{Kh}$ \\
\hline $\begin{array}{c}\text { Saturação de água } \\
\text { conata, Sw }(\%)\end{array}$ & 25 \\
\hline
\end{tabular}

Para realizar as simulações foi utilizado o módulo STARS (Steam, Thermal, and Advanced Processes Reservoir Simulator) da empresa CMG (Computer Modelling Group) na versão 2012.10. Trata-se de um simulador trifásico de múltiplos componentes, que foi desenvolvido com a finalidade de simular recuperações térmicas. 
O solvente utilizado no processo ES-SAGD deste trabalho foi o heptano $\left(\mathrm{C}_{7} \mathrm{H}_{16}\right)$, pois a utilização deste solvente junto com vapor proporciona fator de recuperação de óleo significante.

O modelo de fluidos utilizado foi o "composicional", onde o tratamento matemático além de temperatura e pressão também considera as composições das diversas fases presentes no meio poroso.

O modelo apresenta 8 pseudo-componentes que podem ser observados na Tabela 2. Esta composição de hidrocarbonetos possui uma viscosidade de $656,4 \mathrm{cP}$ a $38^{\circ} \mathrm{C}$.

Tabela 2 - Composição do fluido

\begin{tabular}{|c|c|}
\hline Pseudo-Componentes & Fração molar \\
\hline $\mathrm{CO}_{2}$ & 0,0045 \\
\hline $\mathrm{N}_{2}$ & 0,0027 \\
\hline $\mathrm{C}_{1}-\mathrm{C}_{3}$ & 0,1035 \\
\hline $\mathrm{IC}_{4}-\mathrm{NC}_{5}$ & 0,0032 \\
\hline $\mathrm{C}_{6}-\mathrm{C}_{10}$ & 0,0038 \\
\hline $\mathrm{C}_{11}-\mathrm{C}_{19}$ & 0,1664 \\
\hline $\mathrm{C}_{20}-\mathrm{C}_{39}$ & 0,4615 \\
\hline $\mathrm{C}_{40+}$ & 0,2542 \\
\hline Total & 1 \\
\hline
\end{tabular}

\section{RESULTADOS}

Foram realizadas algumas análises com intuito de verificar o que ocorre dentro do sistema quando se modifica a vazão de injeção de vapor.

A Figura 1 mostra a produção acumulada de óleo para sete casos distintos com vazão de injeção de vapor de 25 t/dia, 50 t/dia, 100 t/dia, 150 t/dia, 180 t/dia, 300 t/dia e 400 t/dia cada, mantendo o percentual de solvente injetado em $10 \%$, a distância vertical entre os poços em $9 \mathrm{~m}$ e a qualidade de vapor em 0,5 , com e sem perda de carga e calor, para uma vazão máxima de produção de $500 \mathrm{~m}^{3} \mathrm{std} / \mathrm{dia}$.

As simulações foram realizadas para diferentes percentuais de solvente injetado onde se obteve o mesmo comportamento das curvas, desta forma optou-se por mostrar o gráfico apenas para $\% \mathrm{~S} 10$. 


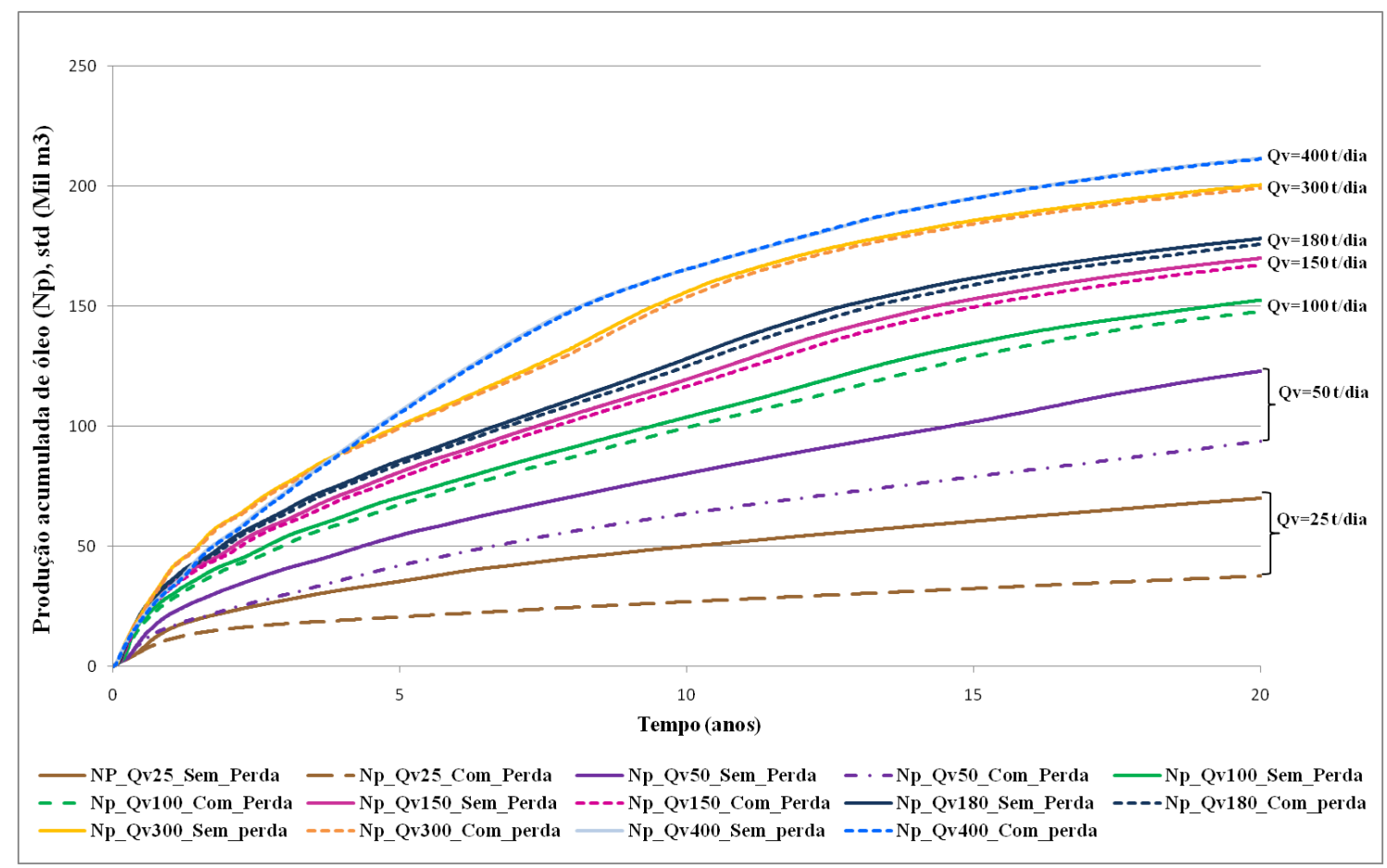

Figura 1- Produção acumulada de óleo versus tempo - comparação entre modelos de vazões diferentes com e sem perda de carga e calor (Dv 9m).

Percebe-se que, em todos os casos o modelo que considera a perda de carga e calor apresenta produção acumulada de óleo inferior ao seu respectivo modelo que desconsidera tais perdas. Essa diferença é mais acentuada quanto menor o valor da vazão de injeção de vapor, ou seja, os modelos com vazão de injeção de vapor de 25 t/dia apresentaram maior diferença na produção acumulada de óleo e os modelos com vazão de injeção de vapor de 400 t/dia foram os de menor diferença obtidos.

Realizou-se a mesma análise para os mesmos sete casos distintos, mantendo o percentual de solvente injetado em $10 \%$, a distância vertical entre os poços em $5 \mathrm{~m}$ e a qualidade de vapor em 0,5 , com e sem perda de carga e calor, para uma vazão máxima de produção de $500 \mathrm{~m}^{3} \mathrm{std} / \mathrm{dia}$. Ou seja, em relação aos modelos da figura anterior, modificou-se apenas a distância vertical entre os poços de $9 \mathrm{~m}$ para $5 \mathrm{~m}$. A Figura 2 mostra as curvas obtidas. 


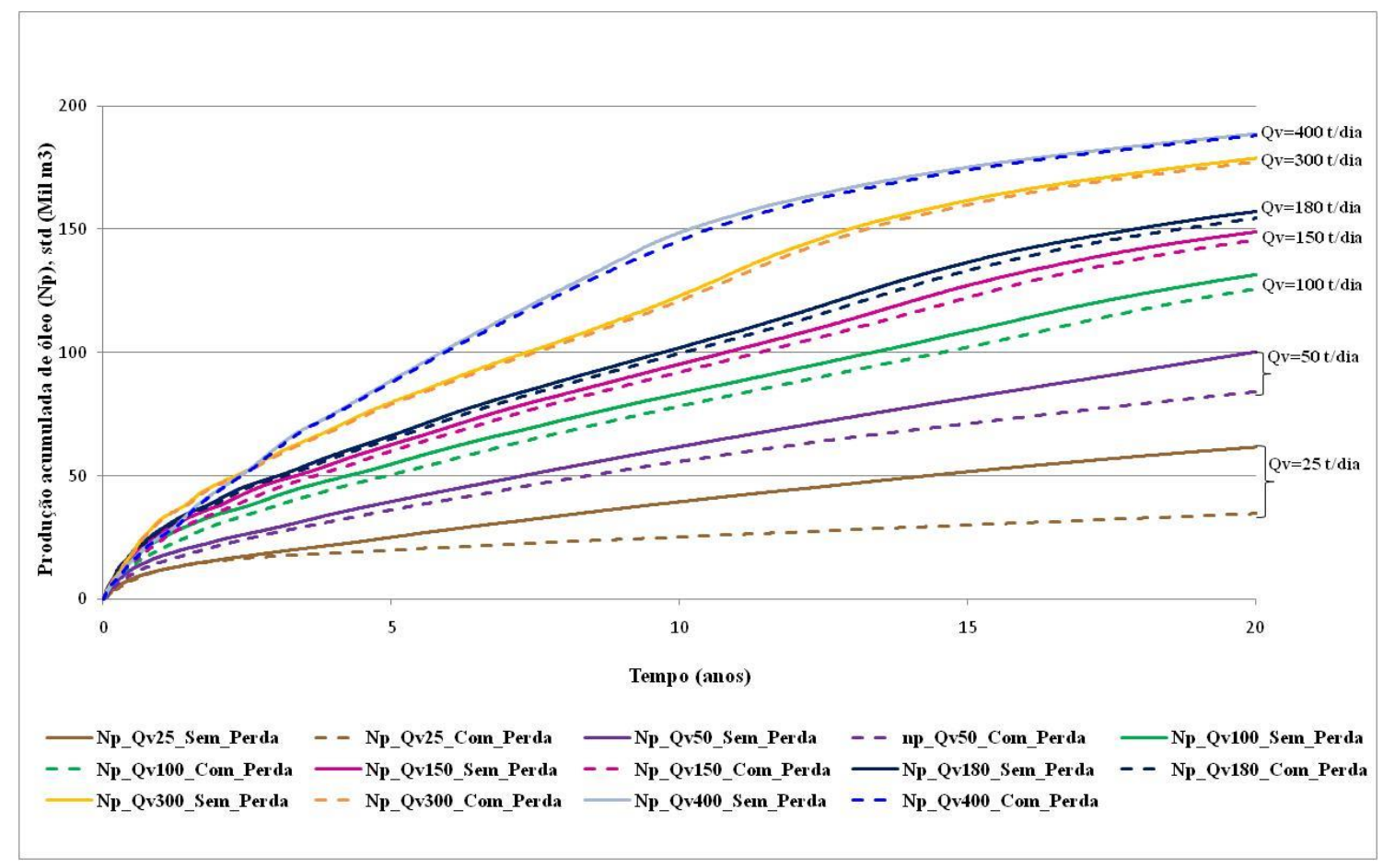

Figura 2 - Produção acumulada de óleo versus tempo - comparação entre modelos de vazões diferentes com e sem perda de carga e calor (Dv 5m).

Observa-se que, as curvas geradas seguem a mesma tendência das obtidas na Figura 1.

Realizou-se através da Equação 1 o cálculo do $\Delta \mathrm{Np}$ entre cada experimento com e sem perda de carga e calor a fim de verificar quantitativamente a variação na $\mathrm{Np}$ obtida quando se considera ou não tais perdas no sistema.

$$
\Delta N p=\frac{\mid N p(\text { Modelosemperda })-N p(\text { Modelocomperda }) \mid}{N p(\text { Modelocomperda })} * 100
$$

Após aplicação da Equação 1 verificou-se que, em concordância com as Figuras 1 e 2 o $\Delta \mathrm{Np}$ possui valores maiores para menor vazão de injeção de vapor. Por exemplo, para a vazão de injeção de vapor de $50 \mathrm{t} /$ dia o $\Delta \mathrm{Np}$ varia entre $6,71 \%$ e $33,41 \%$; para $100 \mathrm{t} /$ dia a variação e de $1,42 \%$ a $5,01 \%$ e para $150 \mathrm{t} / \mathrm{dia}$ os valores estão entre $0,58 \%$ e $2,95 \%$. Também se observou que, o aumento na quantidade de solvente injetado que por conseqüência aumenta a vazão de injeção total (vapor+solvente) reduz os efeitos da perda de carga e calor sobre a Np, para o intervalo analisado. Há apenas dois casos onde os resultados fogem um pouco deste comportamento, podendo ser considerados como erro de convergência do sistema.

Com o objetivo de compreender o que ocorre dentro do sistema durante aplicação do processo ES-SAGD, analisou-se a distribuição de temperatura no reservatório para modelos de baixa e alta vazão de injeção de vapor, ambos com uma vazão máxima de produção de $500 \mathrm{~m}^{3} \mathrm{std} / \mathrm{dia}$. 
A Figura 3 mostra a distribuição de temperatura ao longo dos anos para o modelo com e sem perda de carga e calor, com vazão de injeção de vapor $25 \mathrm{t} /$ dia, percentual de solvente $10 \%$, qualidade de vapor 0,5 e distância vertical entre os poços $9 \mathrm{~m}$.

Observa-se que, no modelo em que a perda de carga e calor é considerada (coluna esquerda) a distribuição da temperatura não é uniforme e o aquecimento do reservatório se torna mais lento. No modelo que desconsidera tais perdas (coluna direita) há uma maior uniformidade da distribuição da temperatura dentro do reservatório e o aquecimento sofre menos limitações ao longo dos anos.
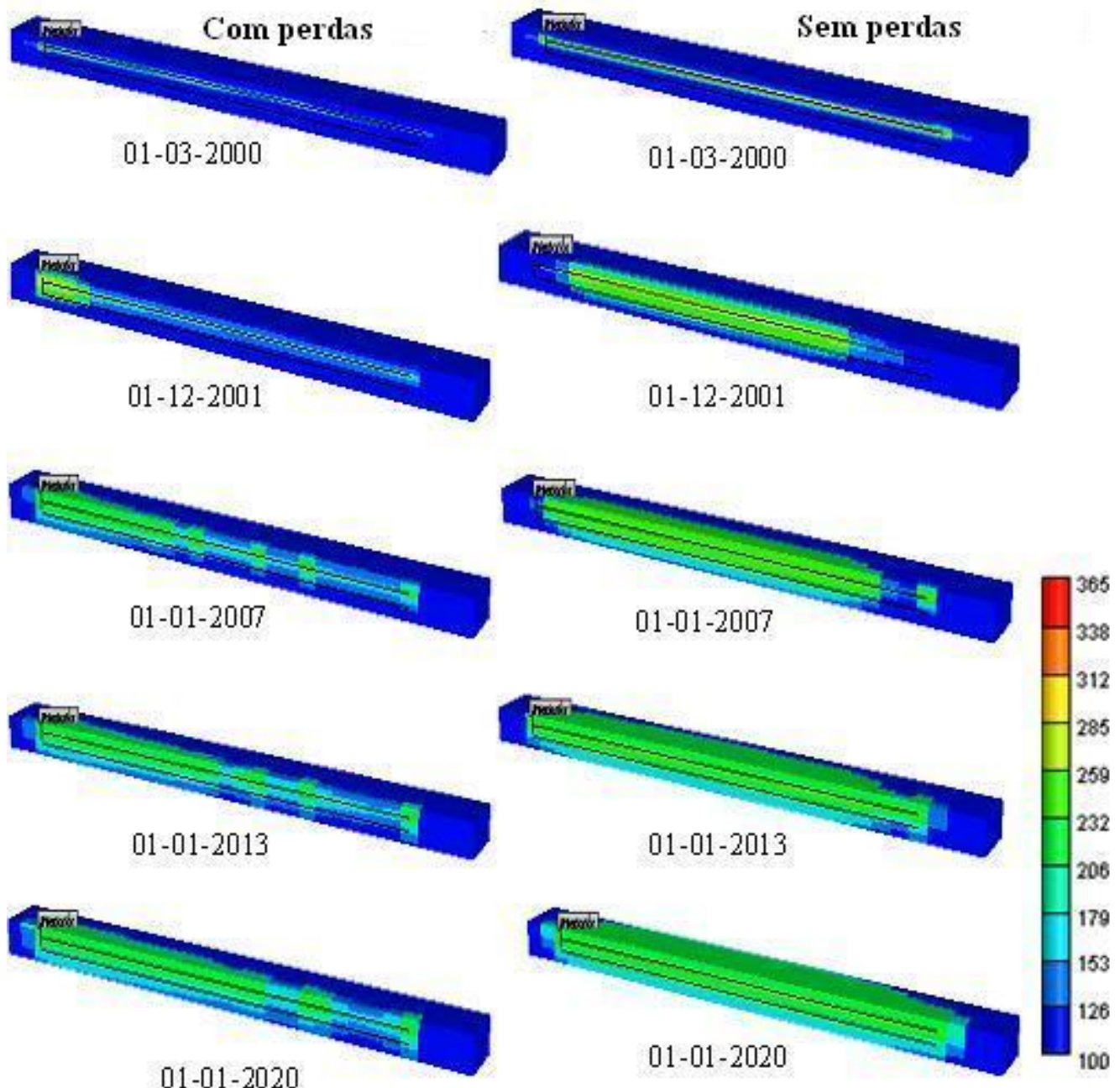

Figura 3 - Comparação da temperatura entre modelo com vazão de injeção de vapor de 25 t/dia, percentual de solvente $10 \%$, qualidade de vapor 0,5 e distância vertical entre os poços $9 \mathrm{~m}$, com e sem perda de carga e calor.

A Figura 4 mostra a distribuição de temperatura ao longo dos anos para o modelo com e sem perda de carga e calor, com vazão de injeção de vapor 180 t/dia, percentual de solvente $10 \%$, qualidade de vapor 0,5 e distância vertical entre os poços $9 \mathrm{~m}$. 
Percebe-se que, no modelo com perdas (coluna esquerda) o aquecimento do reservatório é mais lento em relação ao modelo sem perdas (coluna direita). Porém, ao atingir o período final de produção a diferença entre a área aquecida de cada reservatório não é tão expressiva.

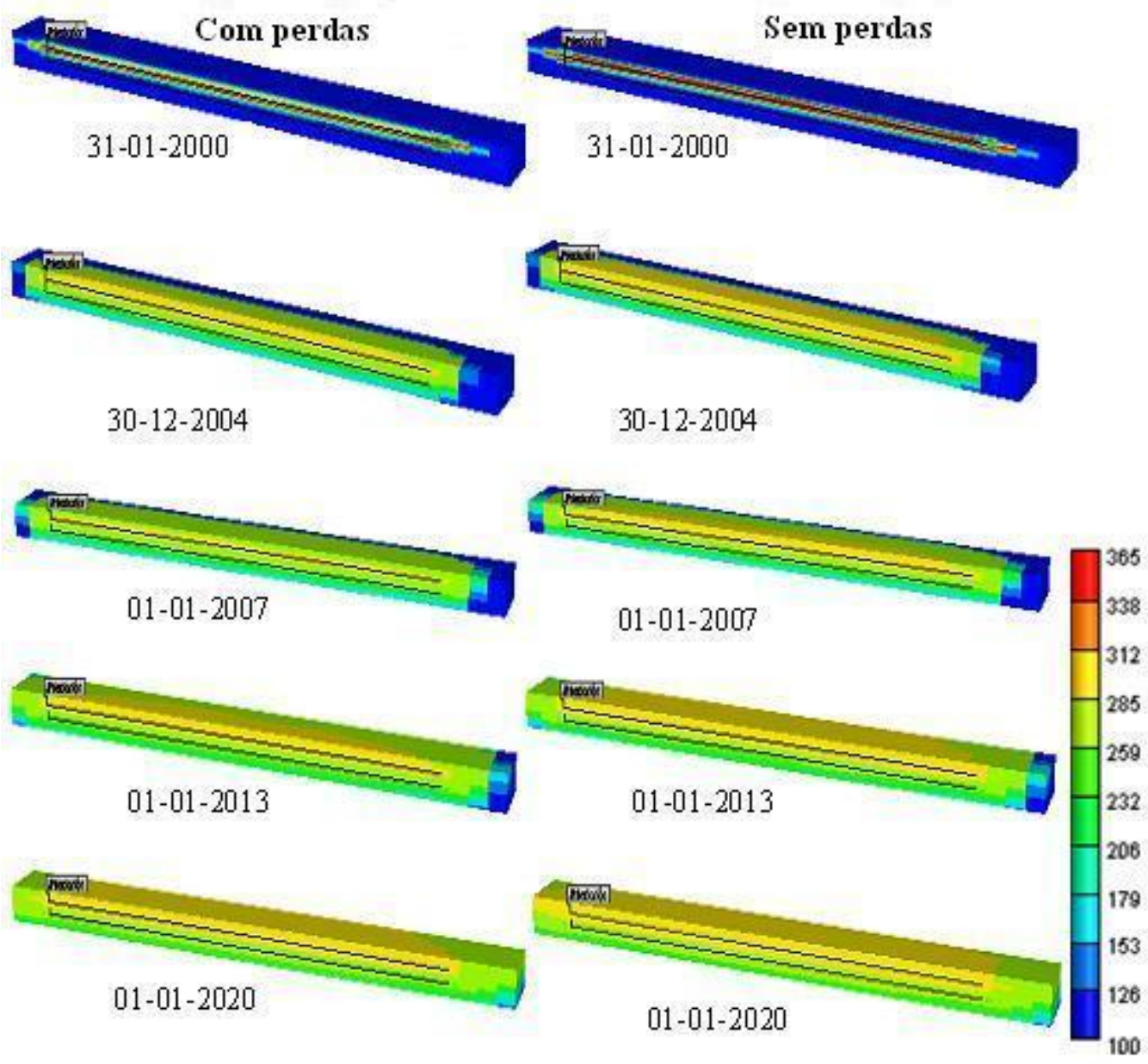

Figura 4 - Comparação da temperatura entre modelo com vazão de injeção de vapor de 180 t/dia, percentual de solvente $10 \%$, qualidade de vapor 0,5 e distância vertical entre os poços $9 \mathrm{~m}$, com e sem perda de carga e calor.

Após análise das Figuras 3 e 4 pode-se entender que, como o modelo com vazão de injeção de vapor de 25 t/dia fornece menos calor ao reservatório do que o modelo com vazão de injeção de vapor de $180 \mathrm{t} / \mathrm{dia}$, tal fato associado às perdas de cargas e calor geram maior dificuldade no aquecimento e produção do óleo presente no reservatório.

Diante do exposto pode-se dizer que, desconsiderar a perda de carga e calor para os modelos com alta vazão de injeção de vapor não afetará muito os resultados obtidos, fato que não se aplica aos modelos com baixa vazão de injeção de vapor. 


\section{CONCLUSÕES}

Verificou-se neste modelo de reservatório que:

- Para todos os casos analisados, o modelo que considera a perda de carga e calor apresenta produção acumulada de óleo inferior ao seu respectivo modelo que desconsidera tais perdas. Essa diferença é mais acentuada quanto menor o valor da vazão de injeção de vapor.

- O modelo com vazão de injeção de vapor de $25 \mathrm{t} /$ dia fornece menos calor ao reservatório do que o modelo com vazão de injeção de vapor de $180 \mathrm{t} / \mathrm{dia}$, tal fato associado às perdas de cargas gera maior dificuldade no aquecimento e produção do óleo presente no reservatório.

- Desconsiderar a perda de carga e calor para os modelos com alta vazão de injeção de vapor não afetará muito os resultados obtidos, fato que não se aplica aos modelos com baixa vazão de injeção de vapor.

\section{AGRADECIMENTOS}

Os autores agradecem ao Programa de Pós Graduação em Ciência e Engenharia de Petróleo (PPGCEP) da Universidade Federal do Rio Grande do Norte (UFRN) pelo suporte recebido para execução do presente trabalho.

A Computer Modeling Group (CMG) pela disponibilidade do simulador computacional.

\section{REFRÊNCIAS}

BARILlaS, J. L. M. "Estudo do Processo de Drenagem Gravitacional de Óleo com Injeção Contínua de Vapor em Poços Horizontais”. Dissertação de Mestrado, Universidade Federal do Rio Grande do Norte, 163 p., Natal, 2005.

BUTLER, R. M. Thermal Recovery of oil and bitumen. Department of Chemical and Petroleum Engineering. Pratice Hall: New Jersey. 1991, 7, 285-358.

DENG, H. et al. Geomechanical and Thermal Simulation of ES-SAGD Process. SPE 148847. Novembro, 2011.

NASR, T.N.; AYODELE, O. R. New Hybrid Steam-Solvent Processes for the Recovery of Heavy Oil and Bitumen. SPE 101717. Novembro, 2006. 